\title{
KERATOCONUS MUST BE DIAGNOSED IN ADVANCE. CASE REPORT AND DISCUSSION OF THE DIAGNOSTIC APPROACH
}

\author{
Dimitar Grupchev, Marin Marinov, Yana Manolova \\ Department of Ophthalmology and Visual Sciences, Faculty of Medicine, \\ Medical University of Varna
}

\begin{abstract}
Performing cataract surgery on a patient with keratoconus is not uncommon. Cataract surgery for such patients does require some additional testing and consideration for the best visual outcomes. Choosing the appropriate lens and lens power can be more challenging. Most patients with keratoconus have high negative spherical aberration and implantation of multifocal intraocular lenses (IOLs) will result in the loss of contrast sensitivity associated with these lenses, which will be magnified by the corneal irregularity. Also, decentration of IOLs relative to the apex of the cone will induce coma and decrease the quality of vision. Our goal was to describe the result after implantation of a multifocal intraocular lens in a patient with forme fruste keratoconus and to show that the preoperative selection of an appropriate IOL in such patients is the key for better visual function.
\end{abstract}

Keywords: keratoconus, multifocal lenses

\section{INTRODUCTION}

Keratoconus is a progressive corneal ectasia, usually with genetic predisposition. It frequently starts at the age of puberty and develops with different rate (1). It often is bilateral, however, it might be significantly asymmetric. Due to a variety of factors and because of its associations with syndromes such as Down syndrome, Alport syndrome, and Ehlers-Danlos, keratoconus patients often develop

Address for correspondence:

Yana Manolova

Specialized Eye Hospital

15 Doyran St.

Varna

e-mail:y.m.manolova@gmail.com

Received: February 27, 2017

Accepted: March 21, 2017 lens opacity and visually disturbing cataracts $(2,3)$. In such cases proper therapeutic decision should be made and that includes selection of the type of surgery, intraocular implants and postoperative optical management $(4,5)$. Considering that keratoconus is endemic for the Balkans, eye care professionals must be alert not only when they operate on patients with established keratoconus but must also screen for forme fruste especially when cataract surgery is performed mainly as a standard refractive procedure.

\section{CASE REPORT}

A 43-year-old man presented at an eye surgical practice with decreased visual acuity of the right eye. During examination a mild sub-capsular cataract was encountered and visual acuity measured 0.5. The patient consented to cataract surgery and insisted on multifocal IOL, as he wanted to be independent from reading glasses. The cataract procedure 
was performed with femtosecond laser and was completely uneventful. During the first postoperative day, the patient complained that his vision had deteriorated. Examination did not highlight any pathology (according to notes) and the patient was told that the brain needed some time to adapt to the situation.

The patient went overseas for a short-term working engagement. After a period of time, he returned and decided to seek a second opinion at the University Eye Clinic - Varna. The examination showed low visual acuity of 0.3 , and significant irregular corneal astigmatism was noted. A clinical examination indicated an uneventful cataract surgery with implantation of refractive multifocal IOL, however Vogt's striae were noted on higher magnification (Fig. 1). Corneal topography was performed to evaluate the nature of the problem. As visible from the keratometric map (Fig. 2), significant nipple keratoconus was prominent in the lower part of the cornea.

The situation was explained to the patient and

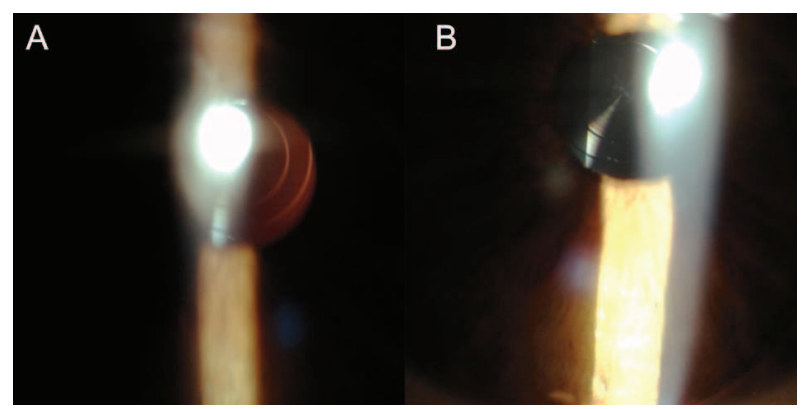

Figure 1. Clinical photography demonstrating multifocal IOL and Vogt's striae in the centre $(A)$ and slightly offcentre lower-temporal opacity (B).

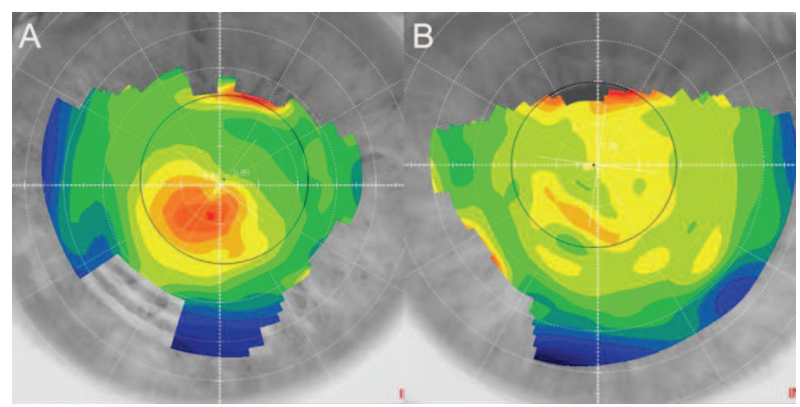

Figure 2. Corneal topography demonstrating keratometric map of the right eye (A) with classic appearance of keratoconus and left eye (B) with suggestion of forme fruste keratoconus.

RGP lens was offered to him. After adaptation to RGP (Fig. 3), visual acuity increased to 0.7 , howev-

er the patient was still having visual disturbances in mesopic and scotopic conditions.

DISCUSSION

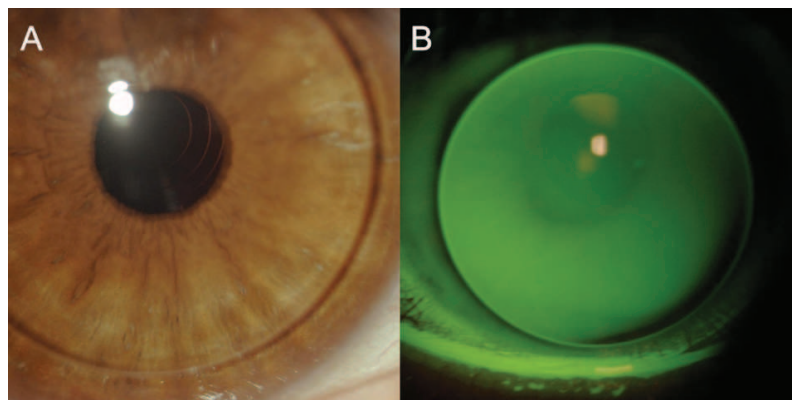

Figure 3. Clinical photography demonstrating adaptation of RGP lens (A) and fluorescein model of the same lens highlighting perfect adaptation and clearance (B).

Keratoconus per se leads to significant microand macro-aberrations (6). Nowadays, the best options for correction are RGP or mini-scleral lenses. Some surgical options, such as intrastromal rings and intracorneal lamellae are also feasible (7). Collagen crosslinking of keratoconic cornea is a promising therapy but it rarely is associated with a significant improvement of the aberrations. This is the reason why some surgeons advocate for combined refractive surgery and crosslinking. Regardless of the controversial results the optical outcomes are usually disappointing and most of those experimental cases require contact lens management of the aberrations. The combination of keratoconus with the multifocal optics of an intraocular lens further complicates the situation with total aberrations (8). First, the optics of multifocal lenses are adapted to certain properties of the normal cornea, and second, the optical system is significantly decentered due to a deviation of the optical axis from the visual axis. These reasons are the basis for the opinion of the multifocal lens producers that keratoconus is an "absolute" contraindication for multifocal IOL implantation.

A refractive surgery algorithm requires screening for keratoconus, however, in standard cataract procedures surgeons do not use topography routinely. Perhaps it should be compulsory when surgery with multifocal lenses is planned because it ensures the patient's vision and the quality of the outcome as well. 
In the reported case we encountered severe keratoconus only of the right cornea, and unfortunately could not say whether it was present prior to surgery or a forme fruste similar to the fellow eye, which developed due to surgical incisions. Importantly, regardless of the excellent visual acuity of 0.9 , the left eye presented a classic topography picture of keratoconus. Unfortunately, postoperative management of the case with RGP lens could not achieve the required visual acuity and more importantly could not improve the quality of vision including contrast sensitivity. Although multifocal IOL exchange is an option due to the relatively long postsurgical period, it has significant risks.

Even if there is no universal solution for this case, it is a good example that sometimes "simple cataract surgery" might be complicated by other conditions. As keratoconus is not rare, but often very subtle, surgeons with limited corneal experience should use topography or similar corneal imaging technique for keratoconus screening when considering multifocal lenses.

\section{REFERENCES}

1. Li X, Yang H, Rabinowitz YS. Keratoconus: classification scheme based on videokeratography and clinical signs. J Cataract Refract Surg, 2009. 35(9): p. 1597-603.

2. Kim JH, et al. Characteristic ocular findings in Asian children with Down syndrome. Eye (Lond), 2002. 16(6): p. 710-4.

3. Carmi E, et al. Ocular complications of atopic dermatitis in children. Acta Derm Venereol, 2006. 86(6): p. 515-7.

4. Arnalich-Montiel F, Alio Del Barrio JL, Alio JL. Corneal surgery in keratoconus: which type, which technique, which outcomes? Eye Vis (Lond), 2016. 3: p. 2.

5. Kolozsvari BL, et al. Correction of irregular and induced regular corneal astigmatism with toric IOL after posterior segment surgery: a case series. 2017. 17(1): p. 3.

6. Kummelil MK, et al. Toric implantable collamer lens for keratoconus. Indian J Ophthalmol, 2013. 61(8): p. 456-60.

7. Lindsay RG, Connell BJ, Snibson GR. Contact lens management of keratoconus in a patient with residual astigmatism resulting from implantation of a toric intraocular lens. Clin Exp Optom, 2013. 96(2): p. 238-41.

8. Ouchi M, Kinoshita S. Implantation of refractive multifocal intraocular lens with a surface-embedded near section for cataract eyes complicated with a coexisting ocular pathology. Eye (Lond), 2015. 29(5): p. 649-55. 